\title{
EFEITOS DO BORO E ZINCO NO TEOR DE CARBOIDRATOS SOLÚVEIS, AMINOÁCIDOS TOTAIS E NO ENRAIZAMENTO DE ESTACAS DE RAMOS DE AMEIXEIRA (Prunus salicina Lindl.) ${ }^{1}$
}

E. KERSTEN

Prof. do Dep. de Fitotecnia da FAEM/UFPEL - CEP: 96015-560-Pelolas, RS. A.A.LUCCIIESI

Prof. do Dep. de Bolanica da ESALQ/USP - Caixa Postal, 9 - CEP: 13418-900-Piracicaba,SP. L.E.GUTIERREZ

Prof. do Dep. de Qutmica da ESALQ/USP - Caixa Posial, 9 - CEP: 13418-900-Piracicaba,SP.

RESUMO: $O$ presente trabalho foi realizado com o objetivo de avaliar o efeito do boro (B) e do zinco (Zn) no teor de carboidratos solúveis, aminoácidos totais e no enraizamento de estacas de ramos dos cultivares Carmesim e Grancuore de ameixeira (Prunus salicina Lindl.). Para a aplicaçao dos tratamentos houve uma fase preliminar que constou da seleção e identificação de quatro filas com vinte plantas cada uma e altemadas com filas de bordadura, que receberam os tratamentos com bórax (B), sulfato de zinco $(\mathrm{Zn})$, bórax mais sulfato de zinco $(\mathrm{B}+\mathrm{Zn})$ e controle. O experimento para enraizamento de estacas de ramos foi realizado em viveiros com nebulização intermitente, no munićpio de Brotas,SP. A coleta de material para avaliação do efeito dos tratamentos com boro, zinco, boro mais zinco e controle, foi executada em maio de 1989, quando parte deste material foi utilizado para determinação de açúcares redutores, açúcares totais e aminościdos totais e outra parte utilizada para 0 enraizamento de estacas. 0 delineamento experimental foi em blocos casualizados. Os resultados obtidos mostraram que o cultivar Carmesim apresentou maior facilidade para enraizar e houve interação entre cultivar com produto (B, $\mathrm{Zn}$, e B $+\mathrm{Zn}$ ) e aumento no teor de aminoácidos totais.

Descritores: enraizamento, ameixeira, Prunus salicina Lindl, boro, zinco, carboidratos solúveis, aminoacidos totais.

\section{EFFECT OF BORON AND ZINC IN SOLUBLE CARBOIIDRATE CONTENT, TOTAL AMINOACIDS AND ROOTING OF PLUM BRANCII CUTTINGS (Prunus salicina Lindl.)}

ABSTRACT: The purpose of this study was to evaluate the effect of boron and zinc on the rooting of branch cuttings of two plum (Prunus salicina Lindl.) cultivars Carmesim and Grancuore. In a previous phase four lines with twenty plants were selected for the application of boron, zinc, boron + zinc and control. The study on the rooting of branch cuttings was conducted at the Brotas County, Săo Paulo State, in a nursery with intermitent artificial mist conditions. Materials were collected in May/89, one part used to analyse reducing sugars, total sugars and total aminoacids and another part used for rooting test of branch cuttings. The results showed that Cammesin had a higher ability for rooting and there was interaction of cultivars with products $(B, Z n$ and $B+Z n)$ and the zinc, boron and boron-zinc increased total aminoacids in the two cultivars.

Key Wonds: rooting, plum, Prunus salicina Lindl., boron, zinc, carbohydrates content, total aminoacids.

\section{INTRODUÇÃO}

A ameixeira (Prunus spp.) é uma espécie frutifera cultivada economicamente no Brasil, principalmente nos Estados de São Paulo, Paraná, Santa Catarina e Rio Grande do Sul. O principal objetivo deste trabalho foi avaliar o efeito do zinco, boro e, zinco mais boro no teor de carboidratos, aminoácidos totais e, relação com o enraizamento de estacas de ramos de dois cultivares de ameixeira.

Segundo SINGH (1981), o zinco faz parte de várias enzimas e pode estar também envolvido na síntese de proteínas e do triptofano. A aplicação de 5 e $10 \mathrm{ppm}$ de zinco, aumentou a concentração de triptofano em grãos de arroz.

Para SKOOG (1940), existe relação entre zinco e auxina. A diminuição de auxina precede

1 Parte da Tese de Doutorado do primeiro Autor - ESALQ/USP - 13418-900-Piracicaba-SP. 1991.

Sci. agric., Piracicaba. 50(1):13-18, fev./maio, 1993 
visivel sintoma de deficiência de zinco. Em plantas mantidas em estágios extremos de deficiência, o aumento em auxina ocorre em poúcos dias após a aplicação de zinco na solução. $O$ zinco não é requerido somente para síntese de auxina, mas para a manutenção de um estado ativo. A falta de zinco acarreta a excessiva destruição de auxina.

Os sintomas de deficiência de boro $\mathrm{em}$ plantas são similares a deficiência em auxina. As funções do boro nas plantas estão relacionadas com a formação de hormônios (EATON, 1940).

Folhas de plantas deficientes em boro apresentam, comparativamente, maiores concentrações de açúcar e amido, contudo a concentração nos ramos é baixa; isto indica que a presença de boro em quantidade adequada é necessária na translocação dos açúcares das folhas para os ramos. Utilizando sacarose e boro juntos e em separado, em solução nutritiva, observou-se estreita dependência na translocação do açúcar em presença de boro; em experimento com pontas de raiz de feijão-de-lima (Phaseolus lunatus L.) e ervilha (Pisum sativum L.) na presença de sacarose mais 5 ppm de boro, comparada com sacarose isolada, constatou-se aumento de 50 para $85 \%$ na respiração, resultando no aumento da sacarose mostrado pela respiração das células (GAUCH \& DUGGER JR., 1953).

Para MURRAY et al. (1957), o boro não tem efeito na emissão de raízes, é essencial para o seu desenvolvimento. Não tem efeito similar aos reguladores vegetais.

Investigação sobre o efeito da deficiência de boro no nível de IAA (ácido indolacético) em girassol (Helianthus anuus L.) feijão (Phaseolus vulgaris L.), milho (Zea mays L.) e plantas de trigo (Triticum sativum L.), indicam a diminuição do contéudo livre de IAA. Raízes de plantas de milho deficientes em boro apresentam altos teores de IAA, comparadas aos de raízes de plantas controle. O teor de IAA aumenta com a deficiência de boro em raízes de trigo. Concluiram que o dano causado por deficiência de boro não está associado com a acumulação de IAA em quantidades tóxicas (SMIRNOV et al., 1967).

TUKEY \& GREEN (1934) em trabalho com ramos de roseiras (Rosa spp.) com um ano de idade, obtidas em substrato com alto e baixo teor de nitrogênio, com $100 \mathrm{~cm}$ de comprimento, foram seccionados da base para a extremidade apical em pedaços com $10 \mathrm{~cm}$ de tamanho. Os resultados apresentaram alta porcentagem de amido em ramos obtidos com suprimento de baixo teor de nitrogênio e menor quantidade de amido em ramos de plantas com alto teor de nitrogênio; em ambos os casos existe um gradiente da base para a extremidade.

MARANGONI et al. (1982), trabalhando na propagação de cerejeira (Phyllanthus spp.) com estacas de ramos de cultivares de difícil enraizamento, tratadas com IBA e colocadas em leito com fundo aquecido, verificaram que estacas tratadas com IBA, diminuiram o peso da máteria seca, carboidratos totais, glicose e sorbitol.

Através do teor de amido determinado no tecido dos ramos com um ano de idade de portaenxertos clonais de ameixeira, GRZYB (1982) concluiu que a habilidade de emissão de raízes aumentou com o aumento do diâmetro dos ramos e o teor de carboidratos nos tecidos.

FACHINELLO et al. (1988) em trabalho realizado com estacas de ramos de porta-enxerto de macieira cultivar MM106, concluiram que os aumentos nos teores de açúcares solúveis $\mathrm{e}$ nitrogênio total na estaca, não foram acompanhados de aumentos no enraizamento. $O$ anelamento é uma prática que aumenta a capacidade das estacas de emitir raízes, devido ao acúmulo de substâncias promotoras do enraizamento (STOLTZ \& HESS, 1966).

Segundo GORDON \& PALEG (1961), os fenóis em condição de oxidação reagem com o triptofano para formar a auxina (ácido indolacético). Também VÁlIO (1979), MALAVOLTA (1980) e SINGH (1981) afirmam que o triptofano é o precursor na formação do ácido indolacético.

\section{MATERIAL E MÉTODOS}

Este trabalho foi executado com ramos de ameixeiras (Prunus salicina Lindl.) em pomar com 5 anos, instalado na fazenda Taperão, em Brotas, SP, com clima classificado como Cfa de acordo com Koeppen, com altitude de $661 \mathrm{~m}$, latitude sul de $22^{\circ} 17^{\prime} 00^{\prime \prime}$ e longitude oeste de $48^{\circ} 08^{\prime} 00^{\prime \prime}$, em Latossolo Vermelho Escuro álico, A moderado, textura média (Quartzisemmentic Haplorthox) de acordo com ALMEIDA et al. (1981).

Avaliou-se o teor de carboidratos (açúcares redutores e açúcares totais), aminoácidos totais e, enraizamento de estacas de ramos, obtidos da porção mediana, de dois cultivares de ameixeira (Prunus salicina Lindl.) Carmesim e Grancuore. Os ramos dos dois cultivares foram obtidos de plantas que receberam em 16/01/1989, $10 \mathrm{~g}$ de bórax $(11,3 \%$ de $\mathrm{B}) ; 10 \mathrm{~g}$ de sulfato de zinco $(67 \%$ 
de $\mathrm{Zn})$ e, $10 \mathrm{~g}$ de bórax mais $10 \mathrm{~g}$ de sulfato de zinco, coletados em 07/05/89.

A extração de carboidratos solúveis foi segundo o método descrito por GUTIERREZ (1975) e a determinacão de açúcares redutores pelo método de Somogy-Nelson, descrito em AMORIM et al. (1982); enquanto os açúcares totais pelo método do fenol-sulfúrico, segundo DUBOIS et al. (1956). A amostra para extração dos carboidratos solúveis constou de 8 porções de ramos com $15 \mathrm{~cm}$ de comprimento, obtidos da parte mediana de ramos coletados de plantas que receberam os tratamentos com bórax (B), sulfato de zinco $(\mathrm{Zn})$, bórax mais sulfato de zinco $(\mathrm{B}+\mathrm{Zn}) \mathrm{e}$, controle. O preparo da amostra constou do corte das porções de ramos em segmentos menores que $0,5 \mathrm{~cm}$ e triturados em moinho do tipo Willey.

O preparo da amostra para determinação dos aminoácidos totais, constou de 8 porções de ramos secos em estufa a $65^{\circ} \mathrm{C}$ por 72 horas, moidas em moinho do tipo Willey com peneira de 20 mesh, mantidas em câmaras com temperatura de $8^{\circ} \mathrm{C}$ para posterior análise foi segundo SILVEIRA:. No enraizamento utilizou-se estacas com $15 \mathrm{~cm}$ de comprimento, da parte mediana de ramos, com um par de folhas, obtidas de plantas tratadas com B, $\mathrm{Zn}, \mathrm{B}+\mathrm{Zn}$ e controle, colocadas em, sacos de polietileno, tendo vermiculita grânulo fino como substrato e mantidas em condições de nebulização intermitente.

O delineamento experimental adotado para os experimentos de enraizamento constou de 12 estacas por tratamento com duas repetições: enquanto que, para açúcares redutores, açúcares totais e amonoácidos totais utilizou-se uma amostra por tratamento e duas repetições.

\section{RESULTADOS E DISCUSSÕES}

Os resultados obtidos neste experimento são apresentados na TABELA 1 , onde se observa efeito acentuado de cultivar na percentagem de estacas enraizadas, onde o cv. Carmesin apresentou $54,16 \%$ de estacas enraizadas e o cv. Grancuore $31,45 \%$. Efeito semelhante também foi mencionado por BARTOLINI et al. (1982) e SHARMA \& AIER (1989) entre os grupos de ameixeiras européias (Prunus salicina L.), sino-japonesas (Prunus salicina, L.) e também por BOLIANI (1986).

- SIL VEIRA, J.A. (USP-Centro de Energia Nuclear na Agricultura). Infornação pessoal, 1989.
O efeito da aplicação de $B, B+Z n, Z n$ e controle na percentagem de estacas enraizadas, são apresentados na TABELA 2, onde se observa que plantas tratadas com zinco apresentaram menor porcentagem de estacas enraizadas (27,91\%), embora não diferissem significatimente do controle com $42,08 \%$. Estacas obtidas de plantas tratadas com boro apresentaram 58,75\% de estacas enraizadas, obtidas de plantas tratadas com zinco. A aplicação de boro mais zinco, aumentou a porcentagem de estacas enraizadas de $27,91 \%$ para $42,50 \%$. Neste experimento o zinco não influiu na percentagem de estacas enraizadas, contrariando princípio estabelecido por SKOOG (1940) da relação entre zinco e auxina e SINGH (1981), entre zinco e triptofano. $O$ efeito de cultivar, produto (B, $\mathrm{B}+\mathrm{Zn}, \mathrm{Zn}$ ) e interação (cultivar $\mathrm{x}$ produto) no teor de açúcares redutores é mostrado na TABELA 3 , onde o cv. Carmesin apresentou maior teor de açúcares redutores que o cv. Grancuore em todos os tratamentos.

TABELA 1 - Efeito de cultivar na percentagem de estacas enraizadas.

\begin{tabular}{cc}
\hline Cultivares & $\%$ de Estacas Enraizadas \\
\hline Carmesin & $54,16 \mathrm{a}$ \\
Grancuore & $31,45 \mathrm{~b}$ \\
\hline
\end{tabular}

Médias seguidas de letras distintas diferem entre si pelo teste de Tukey a $5 \%$ de probabilidade.

C.V. $9,31 \%$

O cv. Carmesin apresentou maior teor de açúcar redutor $(2,65 \mathrm{~g})$, em ramos de plantas tratadas com boro mais zinco, diferindo do controle que apresentou $2,07 \mathrm{~g}$ e dos tratamentos com boro e zinco: enquanto no $\mathrm{cv}$. Grancuore não houve diferença entre os tratamentos, a diferença entre cultivar, talvez seja devido a característica genética.

O efeito da aplicação de $B, B+Z n$ no teor de açúcares totais ć mostrado na TABELA 4, onde se observa que só o cv. Carmesin apresentou diferença significativa entre os tratamentos sendo que com boro mais zinco apresentou $6,44 \mathrm{~g}$, que não diferiu do controle com $5,65 \mathrm{~g}$, mas diferiu dos tratamentos com boro e com zinco. É possível que este efeito pouco pronunciado no teor de açúcares totais para os dois cultivares, seja devido ao fato de estarem próximos do limite máximo de 
TABELA 2 - Efeito de B, B + Zn e Zn na percentagem de estacas enraizadas.

\begin{tabular}{ccccc}
\hline \hline Produto & Boro & Boro + Zinco & Zinco & Controle \\
\hline $\begin{array}{c}\text { \% de estacas } \\
\text { enraizadas }\end{array}$ & $58,75 \mathrm{a}$ & $42,50 \mathrm{ab}$ & $27,91 \mathrm{~b}$ & $42,08 \mathrm{ab}$ \\
\hline C.V. $9,31 \%$ & & & \\
\hline \hline
\end{tabular}

Médias na mesma linha seguidas de letras distintas diferem entre si pelo teste de Tukey a $5 \%$ de probabilidade.

TABELA 3 - Efeito da interação cultivar x produto no teor de açúcares redutores, em $\mathrm{g} / 100 \mathrm{~g}$ de $\mathrm{m} . \mathrm{s}$.

\begin{tabular}{ccccc}
\hline \hline & \multicolumn{5}{c}{ P R O D U T O } \\
\cline { 2 - 5 } Cultivar & Boro & Boro + Zinco & Zinco & Controle \\
\hline Carmesim & $2,13 \mathrm{~b}$ & $2,65 \mathrm{a}$ & $1,91 \mathrm{~b}$ & $2,07 \mathrm{~b}$ \\
Grancuore & $1,55 \mathrm{a}$ & $1,53 \mathrm{a}$ & $1,38 \mathrm{a}$ & $1,59 \mathrm{a}$ \\
\hline C.V.6,72\% & & & \\
\hline \hline
\end{tabular}

Médias na mesma linha seguidas de letras distintas diferem entre si pelo teste de Turkey a $5 \%$ de probabilidade.

TABELA 4 - Efeito da interação cultivar $x$ produto no teor de açúcares totais, em $\mathrm{g} / 100 \mathrm{~g}$ de m.s.

\begin{tabular}{ccccc}
\hline & & P R O D U T O & \\
\cline { 2 - 5 } Cultivar & Boro & Boro + Zinco & Zinco & Controle \\
\hline Carmesim & $5,21 \mathrm{~b}$ & $6,44 \mathrm{a}$ & $4,95 \mathrm{~b}$ & $5,65 \mathrm{ab}$ \\
Grancuore & $5,46 \mathrm{a}$ & $4,74 \mathrm{a}$ & $4,74 \mathrm{a}$ & $5,20 \mathrm{a}$ \\
\hline C.V. 7,77\% & & & \\
\hline \hline
\end{tabular}

Médias na mesma linha seguidas de letras distintas diferem entre si pelo teste de Tukey a $5 \%$ de probabilidade.

acúmulo, haja visto que a coleta deste material ocorreu em maio.

O efeito de cultivar e produto (aplicação de $\mathrm{B}, \mathrm{B}+\mathrm{Zn}$ e $\mathrm{Zn}$ ) no teor de aminoácidos totais é mostrado na TABELA 5 , onde se observa no $\mathrm{cv}$. Carmesim os maiores valores nos tratamentos que receberam zinco e boro mais zinco, que não diferiram entre si, mas diferiram do tratamento com boro e controle. Enquanto, no cv Grancuore os tratamentos com zinco e boro mais zinco diferiram entre si e do controle, apresentando uma variação de 0,065 a 0,111 microgramas. Estes 
TABELA 5 - Efeito da interação cultivar x produto no teor de aminoácidos totais, em microgramas $/ 100 \mathrm{mg}$ de m.s.

\begin{tabular}{ccccc}
\hline & \multicolumn{5}{c}{ P R O D U T O } \\
\cline { 2 - 5 } Cultivar & Boro & Boro + Zinco & Zinco & Controle \\
\hline Carmesim & $0,080 \mathrm{~b}$ & $0,125 \mathrm{a}$ & $0,123 \mathrm{a}$ & $0,072 \mathrm{c}$ \\
Grancuore & $0,104 \mathrm{~b}$ & $0,111 \mathrm{a}$ & $0,092 \mathrm{c}$ & $0,065 \mathrm{~d}$ \\
\hline C.V. 1,64\% & & & \\
\hline \hline
\end{tabular}

Médias na mesma linha seguidas de letras distintas diferem entre si pelo teste de Tukey a $5 \%$ de probabilidade.

resultados estão de acordo com resultados obtidos por SINGH (1981), com aplicação de zinco em plantas de arroz que aumentou a concentração de triptofano nos grãos.

\section{CONCLUSÕES}

- o cv. Carmesim apresentou maior facilidade para enraizar do que o cv. Grancuore;

- a aplicação de bórax ou sulfato de zinco não interferiu na percentagem de estacas enraizadas;

- houve interacão de cultivar com bórax, sulfato de zinco, em scparado ou conjuntamente, no teor de açúcares redutores, açúcares totais e aminoácidos totais: bórax mais sulfato de zinco, no teor de açúcares redutores, açúcares totais e aminoácidos totais;

- a aplicação de bórax e sulfato de zinco, em separado ou conjuntamente aumentou o teor de aminoácidos totais.

\section{REFERÊNCIAS BIBLIOGRAFICAS}

ALMEIDA, C.F.L. de; OLIVEIRA, J.B, de; PRADO, $H$. do. Levantamento pedologico semi-detalhado do Estado de São Paulo; quadricula de Brotas. São Paulo: Secr. Agricultura e Abastecimento, 1981. (Mapa).

AMORIM, H.V.; ZAGO, E.A.; OLIVEIRA, A.J. Novos métodos para o controle da fermentação alcóolica. São Paulo. Sociedade Brasileira de Microbiologia. 1982, 58p.
BARTOLINI, G.; ROSELLI, G.; MESSER, C. Richerche sulla propagazione del susino per talea di ramo: 4-tecniche de moltiplicazione ed incrementi di crescita di cultivar autoradicate di Prunus domestica e Prunus salicina. Revista della Ortoflorofrutticoltura Italiana, Florence, v.66, n.2, p.161-71, 1982.

BOLIANI, C.A. Efeitos do estiolamento basal, da juvenilidade e do uso de um regulador vegetal no enraizamento de estacas de raízes e de ramos herbáceos de algumas espécies frutíferas. Piracicaba, 1986. 129 p. (Dissertação de Mestrado). Escola Superior de Agricultura "Luiz de Queiroz", Universidade de São Paulo.

DUBOIS, M.; GILBS, K.A.; HAMILTON, J.K.; REBENS, P.A.; SMITH, F. Colorimetric method for determination of sugars and related substances. Analytical Chemistry, Washington, v.28, p.350-356, 1956.

EATON, F.M. Interrelations in the effects of boron and indolacelic acid on plant growth. Botanical Gazette, Chicago, v.101, p.700-705, 1940.

FACHINELLO, J.C.; LUCCHESI, A.A.; GUTIERREZ, L.E. Influência do anelamento na nutrição e no enraizamento de estacas lenhosas do porta-enxerto 'Malling-merton 106'. Pesquisa Agropecuária Brasileira, Brasilia, v.23, n.9, p.1025-1031, 1988.

GAUCH, H.G.; DUGGER JR., W.M. The role boron in the translocation of sucrose. Plant Physiology, Rockville, v.28, p.457-466, 1953.

GORDON, S.A.; PALEG, L.G. Formation of auxin from tryptophan through action of polyphenols. Plant Physiology, Rockville, v.36, p.838-845, 1961. 
GRZYB, Z.S. Growth and rooting of Brompton plum F 12/1 cherry and M 26 clonal rootstocks: III. The relationship between the starch content in shoots of the plum clonal rootstocks and their ability to form adventitious roots. Fruit Science Reports, Skierniewice, v.9, n.1, p.13-19, 1982.

GUTIERREZ,L.E. Identificação de carboidratos e ácidos orgânicos em quatro variedades de capim elefante (Pennisetum purpureum, Schum), colhidas em três estágios de maturidade. Piracicaba, 1975. 103 p. (Dissertação de Mestrado). Escola Superior de Agricultura "Luiz de Queiroz", Universidade de São Paulo.

MALAVOLTA, E. Elementos de nutrição mineral de plantas. São Paulo, Agronômica Ceres, 1980. 251p.

MARANGONI, B.; CRISTOFERI, G.; ROSSI, F. Rooting ability and carbohydrate metabolism in hardwood cherry cuttings. Rivista della Ortoflorofrutticoltura Italiana, Florence, v.66, p.437-444, 1982.

MURRAY, H.R.; TAPER, C.D.; PICKUP, T.; NUSSEY, A.W. Boron nutrition of softwood cuttings of geranium and currant relation to root development, Proceedings of the American Society for IIorticultural Science, College Park, v.69, p 498-501, 1957.

SHARMA, S.D.; AIER, N.B. Seasonal rooting behavior of cuttings of plum cultivar as influenced by IBA treatments Scientia IIorticulturae, Amsterdan, v 40, p.297-303, 1989.
SINGH, M. Effect of zinc, phosphorus and nitrogen on triptophan concentration in rice grains grown on limed and unlimed soils. Plant and Soil, Dordrecht, v.62, n.2, p.305-308, 1981.

SKOOG, F. Relationships between zine and auxin in the growth of higher plants. American Journal of Botany, New York, v.27, p.939-950, 1940

SMIRNOV, S.Yu; KRUPNIKOVA, T.A.; SHKOL'NIK, M.Ya. Content of IAA in plants with differen sensitivity to boron deficits. Soviet Plant Physiology, New York, v.24, p.270-276, 1967.

STOLTZ, L.P.; HESS, C.E. The effect of girdling upon root initiation: Carbohydrates and aminoacids. Proceedings of the American Society for Horticultural Science, College Park, v.89, p.734-743, 1966.

TUKEY, H.B.; GREEN, E.E.L. Gradient composition of rose shoots from tip to base. Plant Physiology, Rockville, v.9, p.157-163, 1934.

VÁLIO, I.F.M. Auxinas. In: FERRI, M.G., (Coord.). Fisiologia vegetal. São Paulo, EPU, EDUSP, 1979. v 2, p. 39-72.

Trabalho entregue para publicaçāo em 13.02.92

Trabalho aprovado para publicação em 04.09 .92 